\title{
3Dexterity: Finding your place in a 3-armed world
}

\author{
Alexandre Audinot*1, Diane Dewez*1, Gwendal Fouché*1, Rebecca Fribourg*1, Thomas Howard *1, Flavien Lécuyer ${ }^{* 1}$, Tiffany \\ Luong ${ }^{* 1,2}$, Victor Mercado ${ }^{* 1}$, Adrien Reuzeau*1, Thomas Rinnert*1, Guillaume Vailland*1 ${ }^{* 1}$, and Ferran Argelaguet*1 \\ ${ }^{1}$ Univ Rennes, Inria, INSA, CNRS, IRISA, Rennes, France \\ ${ }^{2}$ IRT $\mathrm{b}<>$ com, Cesson-Sevigne, France
}

\begin{abstract}
In the context of the IEEE VR 2020 3DUI Contest entitled "Embodiment for the Difference", this paper presents a VR application to highlight the challenges that people with physical disabilities face on their daily life. Two-armed users are placed in a world where people normally have three arms, making them effectively physically disabled. The scenario takes the user through the process of struggling with everyday interactions (designed for humans with three arms), then receiving a third arm prosthesis and thus recovering some level of autonomy. The experience is intended to generate a sense of difference and empathy for physically disabled persons.
\end{abstract}

\section{INTRODUCTION}

In the presented virtual reality experience, users (having two arms) inhabit a world in which humans have evolved to have three arms (see Figure 1). As such, users will encounter difficulties to interact with their environment due to the differences between their own body (only two arms) and the "standard" three-armed body that makes up the norm in this fictional world. In this experience, the world and tools are designed for people with three arms which puts users in an uncomfortable context. The experience is divided in three phases. In the first phase of the experience, users will have to approach the world and attempt to achieve tasks designed for tri-manual control using only their two arms. The goal is to show frustration, inconvenience as well as giving users a feeling of inadequacy and social exclusion. Furthermore, it reflects everyday challenges that face people with physical disabilities in daily tasks and tools designed for people with a wider range of physical capabilities. In the second phase, users are given a third arm prosthesis intended to compensate for their handicap, thus recovering some level of autonomy in interaction with their environment. However, the prosthesis is both imperfect and complex to master, highlighting real-world issues with current prosthetic solutions for people with disabilities. In the third phase, users can perform the same interactions as in the first phase but using the prosthesis to achieve them.

\section{Controlling a Third Arm}

In this demonstration, users will need to control a third arm prosthesis. In the literature, there exist a number of works that have already explored and evaluated the control of additional arms. For example, in a virtual reality context, Laha et al. [1] proposed and evaluated three different methods to control a third arm (bimanual, unimanual, and head-control). Another interesting example is the work from Steptoe et al. [4] which studied embodiment within an avatar having a tail (hip control). Finally, in a real-world context, Saraiji et al. [3] proposed a robotic system composed of two robotic arms attached to users' back and controlled thanks to the feet and the knees. Parietti et al. [2] propose a similar robotic system with a different approach to control, where the robot autonomously adjusts its state based on interaction positions and forces and a model of the
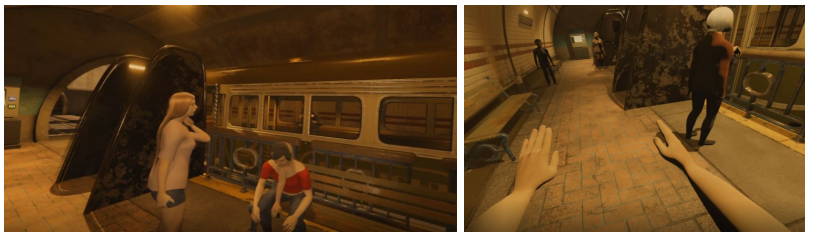

Figure 1: Left: Virtual environment representing a subway gate and populated with persons having three arms. Right: First-person perspective on user's only two arms.

task. From the different examples in the literature, direct control of additional limbs through coupling with a user's head, limbs, hips or individual muscle's state appears to be the most used method. In this experience we propose two different control mechanisms: a novel command-based control and a continuous control.

\subsection{Command-based Control}

In the discrete control mode, users' gaze is used to define interaction targets for the third arm. Then, users can issue voice commands to trigger specific actions with the gaze-designated target. Initially, the third arm prosthesis is in an "inactive"/"idle" state. By saying "activate", users switch on the prosthesis, putting it into an "activewaiting" state where targets can be designated using the gaze and actions can be triggered. Users then have the choice between several actions: "go" (extends the arm towards the target), "back" (retracts the arm), "push" (pushes against the target), "grasp" (grasps the target) and "release" (releases a grasped object). Once an action is triggered, the prosthesis switches into an "active-doing" state where no further action can be triggered. Upon action completion, the prosthesis returns into the "active-waiting" state. The key advantages of this control mode lie in the fact that both users' hands are free for simultaneous interaction with the environment. However, this control scheme can become limited for complex movements and interactions not initially planned.

\subsection{Continuous Control}

Users can switch to a continuous control mode for the arm by using a dedicated voice command. In this mode, users hold down the trigger in their left or right hand to designate that hand as a guide for the prosthesis. This allows users to "drag" the third arm prosthesis to a desired position or through a desired sequence of movements. This control mode provides an intuitive way to move the prosthesis while also providing great freedom of movement and interactions. However, one of users' hands is always occupied by the prosthesis' control during the movement phase, hindering some movement combinations.

\section{Demo apparatus}

The experience uses a HTC Vive Pro Eye virtual reality headset, which integrates Tobii eye tracking technology to enable tracking of users' gaze. We use the headset's built-in microphone to register voice control commands. Voice input is parsed locally on the PC running the demo using the Windows Speech Recognition platform, 
with parsed words being compared to a previously defined dictionary of command words. An experimenter equips users with a chest-strap holding the haptic feedback device while users are being equipped with the virtual prosthetic device in the doctor's office scene. Vibrotactile feedback indicating prosthesis movement and collisions with the environment is provided to the chest via a pair of Actronika HFBA 9532 voice-coil actuators driven by a Tactronik standard board connected to the PC running the demo via USB.

\section{SCENARIO AND INTERACTIONS}

As a two-armed human, users face difficulties in simple daily tasks, with interfaces designed for tri-manual interactions. The first scene takes place in a subway station. Users intend to take the subway to get to a doctor's appointment. There, they will receive a third arm prosthesis and learn how to use it before returning home, now capable of using devices designed for three-handed people.

\subsection{Before receiving a prosthesis}

\subsubsection{First task: Reloading the transport card}

Users need to reload their transport card at the ticket machine before entering the subway (see Figure 2 left). The particularity of this task is that the ticket machine is designed in such a way that users have to hold both their transportation card and their credit card up against the respective card readers, all the while pressing another button to make the adequate selections. Once the selection is made, the presence of the credit card against the reader triggers the payment and order confirmation. If the order is not confirmed in time, it is canceled and users must repeat the process. As a three-armed user, each hand can perform a sub-task (holding one of the cards against a reader, pressing the button) simultaneously so as to easily achieve the task. Since the ticket machine interface expects such users, the countdown before cancellation is quite short. Therefore, the task is achievable as a two-armed user but proves very difficult. Failures cause frustration to users, amplified by background sounds of discontent from other people waiting in line to use the machine. Once the card is recharged, users can move to the train access gates.
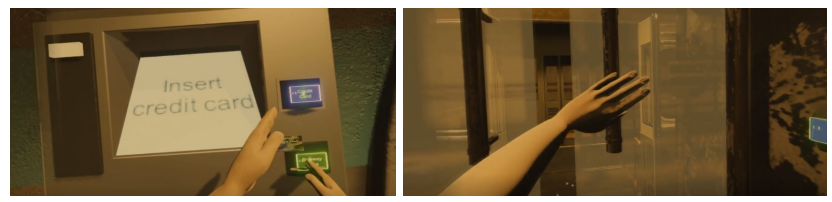

Figure 2: The main tasks to perform in the virtual environment. Left: Reloading the transport card on a dedicated machine. Right: Crossing the gate by opening its doors in order to enter the subway carriage.

\subsubsection{Second task: Crossing the gate}

For the next task, users must cross the gate, once again designed for tri-manual operation (see Figure 2 right). The transportation card must be maintained on the card reader, unlocking both doors that can then be individually pushed. Two-armed users will only be able to push one door, creating an opening too narrow for them to go through. Consequently, this task is impossible to achieve. Users can
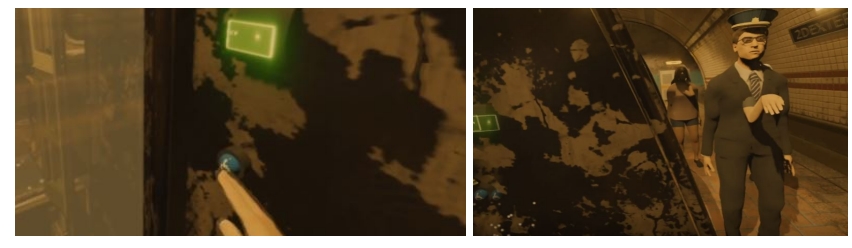

Figure 3: The call for help. Left: User's hand reaching for the help button. Right: Security officer coming to activate the automatic opening. call for help or use the button with a handicap logo in order to have a security officer activate the automatic opening, stressing users' lack of autonomy (see Figure 3). If users are too slow, the train can leave before they cross the doors, causing additional frustration. Once users enter the train, they are fast-forwarded to the medical office.

\subsection{Getting the third arm prosthesis}

In a medical office, users are introduced to the third arm prosthesis and are taught on how to use it. After a talk with a virtual doctor, a black transition is displayed when users are equipped with the prosthesis. At that moment, the experimenter equips users with the haptic device, while sound effects are played to simulate an operation. The prosthesis controls are then explained through a speech tutorial provided by the virtual doctor, followed by a minigame. In the mini-game, users have to reproduce a sequence of notes on three drums placed in front of them (see Figure 4).
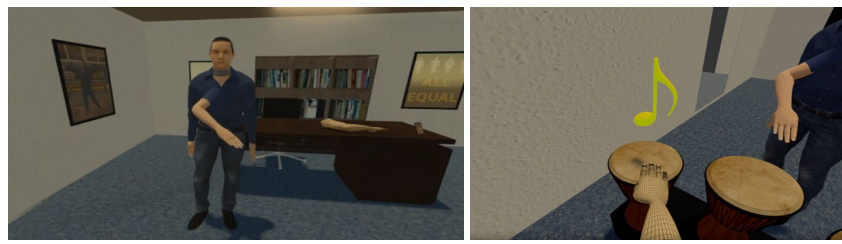

Figure 4: The doctor's office. Left: Doctor welcoming the user. Right: The bongos used as a tutorial for controlling the third arm.

\subsection{After receiving the prosthesis}

Now that users have a third arm, they once again face the same tasks as before in the subway station, and see the differences with their first attempt.To reload their transport card, users now simply have to use their prosthesis to hold one of the cards against the associated reader, freeing up one hand to easily go through the rest of the process. Proceeding to the subway gate, they no longer need to ask for help to get through the doors as the prosthesis can be used to push against one of the doors once unlocked. Passing through the doors ends the experiment, with a message telling the user to remove the headset.

\section{Conclusion}

This paper presents an original scenario for a 3DUI contest that plunges users into an uncomfortable experience in a world which is not adapted to their morphology. Virtual agents in the simulation all have an additional third arm, which users are missing. This makes interactions difficult for users, maximizing frustration, providing a sense of difference and generating empathy for people with physical disabilities. In the end, users are given a prosthesis controlled by voice or arm movements. The prosthesis' imperfection and control complexity add to the awareness or real-world issues of such aids.

\section{REFERENCES}

[1] B. Laha, J. N. Bailenson, A. S. Won, and J. O. Bailey. Evaluating control schemes for the third arm of an avatar. PRESENCE: Teleoperators and Virtual Environments, 25(2):129-147, 2016.

[2] F. Parietti and H. H. Asada. Supernumerary robotic limbs for aircraft fuselage assembly: body stabilization and guidance by bracing. In 2014 IEEE International Conference on Robotics and Automation (ICRA), pp. 1176-1183. IEEE, 2014.

[3] M. Saraiji, T. Sasaki, K. Kunze, K. Minamizawa, and M. Inami. Metaarms: Body remapping using feet-controlled artificial arms. In The 31 st Annual ACM Symposium on User Interface Software and Technology, pp. 65-74. ACM, 2018.

[4] W. Steptoe, A. Steed, and M. Slater. Human tails: Ownership and control of extended humanoid avatars. IEEE Transactions on Visualization and Computer Graphics, 19(4):583-590, April 2013. doi: 10.1109/TVCG. 2013.32 